Pacific Journal of Mathematics

A HAUSDORFF-YOUNG THEOREM FOR 


\section{A HAUSDORFF-YOUNG THEOREM FOR \\ REARRANGEMENT-INVARIANT SPACES}

\section{Colin BenNetT}

The classical Hausdorff-Young theorem is extended to the setting of rearrangement-invariant spaces. More precisely, if $1 \leqq p \leqq 2, p^{-1}+q^{-1}=1$, and if $X$ is a rearrangement-invariant space on the circle $T$ with indices equal to $p^{-1}$, it is shown that there is a rearrangement-invariant space $\hat{X}$ on the integers $Z$ with indices equal to $q^{-1}$ such that the Fourier transform is a bounded linear operator from $X$ into $\hat{X}$. Conversely, for any rearrangement-invariant space $Y$ on $Z$ with indices equal to $q^{-1}, 2<q \leqq \infty$, there is a rearrangement-invariant space $\check{Y}$ on $T$ with indices equal to $p^{-1}$ such that $\mathscr{T}$ is bounded from $\breve{Y}$ into $Y$.

Analogous results for other groups are indicated and examples are discussed when $X$ is $L^{p}$ or a Lorentz space $L^{p r}$.

By $L^{p}=L^{p}(T)$ we denote the usual Lebesgue space on the unit circle $T$, and by $l^{p}=l^{p}(Z)$ the corresponding space on the integers $Z$. The index conjugate to $p$ will always be denoted by $q$ so that $p^{-1}+q^{-1}=1$. The Fourier transform $\mathscr{T}$ defined by

$$
(\mathscr{T} f)(n)=\hat{f}(n)=\frac{1}{2 \pi} \int_{0}^{2 \pi} f(\theta) e^{-i n \theta} d \theta
$$

is a bounded linear operator from $L^{1}$ into $l^{\infty}$ and from $L^{2}$ into $l^{2}$ so by the M. Riesz-Thorin interpolation theorem ([9], p. 95), $\mathscr{T}$ is bounded also from $L^{p}$ into $l^{q}$ whenever $1<p<2$. This is the assertion of the classical Hausdorff-Young theorem ([9], p. 101).

Hardy and Littlewood ([5]; [9], p. 109) showed that $\mathscr{T}$ is bounded from $L^{p}, 1<p<2$, into $l_{p}^{q}$, the "weighted" Lebesgue space of all sequences $\left\{c_{n}\right\}$ for which

$$
\|c\|_{l_{p}^{q}}=\left\{\sum_{-\infty}^{\infty}\left|c_{n}\right| p(1+|n|)^{p-2}\right\}^{1 / p}=\left\{\sum_{-\infty}^{\infty}\left[(1+|n|)^{1 / q}\left|c_{n}\right|\right]^{p}(1+|n|)^{-1}\right\}^{1 / p}
$$

is finite; since $l_{p}{ }^{q} \subseteq l^{q}$, as a simple computation shows, their result improves on that of Hausdorff and Young. A still sharper version, again due to Hardy and Littlewood ([6]; [9], p. 123), is based upon the observation that even the (symmetric) decreasing rearrangement of the sequence $\{\hat{f}(n)\}$ belongs to $l_{p}^{q}$, or, what amounts to the same thing, $\mathscr{T} f$ belongs to the Lorentz space $l^{q p}$ (cf. [3], [4], and [9] for the precise statements and definitions). Thus $\mathscr{T}$ is a bounded 
linear operator from $L^{p}$ into $l^{q p}$ whenever $1<p<2$. More generally, the recent interpolation theorem of Calderón ([3], p. 293) shows that $\mathscr{T}$ is bounded from $L^{p r}$ into $l^{q r}, 1<p<2,1 \leqq r \leqq \infty$, the HardyLittlewood results thus being contained in the special case $r=p$.

It is our intention in this paper to extend the above results to the setting of arbitrary rearrangement-invariant spaces. Intrinsic interest apart, the need for such a theorem arises naturally in problems concerning the ideal structure of Lipschitz subalgebras of rearrangement-invariant spaces (cf. [1]). Our main results, in which the $L^{p}$ spaces are replaced by rearrangement-invariant spaces with indices (cf. [2]) equal to $p^{-1}$, are as follows:

THEOREM A. Let $X$ be a rearrangement-invariant space on $T$ with indices $\left(p^{-1}, p^{-1}\right), 1 \leqq p \leqq 2$. Then there is a rearrangementinvariant space $\hat{X}$ on $Z$ with indices $\left(q^{-1}, q^{-1}\right), p^{-1}+q^{-1}=1$, such that $\mathscr{T}$ is a bounded linear operator from $X$ into $\hat{X}$.

THEOREM B. Let $Y$ be a rearrangement-invariant space on $Z$ with indices $\left(q^{-1}, q^{-1}\right), 2<q \leqq \infty$. Then there is a rearrangement-invariant space $\check{Y}$ on $T$ with indices $\left(p^{-1}, p^{-1}\right), p^{-1}+q^{-1}=1$, such that $\mathscr{T}$ is a bounded linear operator from $\breve{Y}$ into $Y$.

The construction of the spaces $\hat{X}, \check{Y}$ depends crucially on the properties of the maximal operator $S=S(\sigma)$ of Calderón ([3]), and the proof of the boundedness of $\mathscr{T}$ follows from the corresponding interpolation theory. One advantage of this type of proof is that it is then easy to see that Theorems A and B extend to transforms given by arbitrary uniformly bounded orthonormal systems as in earlier results of F. Riesz and Paley ([9], pp. 102, 121). Our results extend to the Fourier transform defined on $Z$ (the "duals" to Theorems $\mathrm{A}, \mathrm{B}$ ) and the real line $R$, and to more general groups (although the theory presented here requires that the Haar measure be always $\sigma$-finite); we shall not aim for this level of generality. Examples are discussed which show that Theorems A and B contain as special cases the results of Hardy-Littlewood and Calderón mentioned above.

2. Rearrangement-invariant spaces. This section contains a brief synopsis of results from the theory of rearrangement-invariant spaces required later. We shall assume that the reader is familiar with the material in the paper of Boyd ([2]) whose notation we shall by and large adhere to; for further details see [4], [7], and [8].

Thus $(\Omega, \mathscr{T}, \mu)$ will denote a totally $\sigma$-finite measure space, $\mu \geqq 0$, satisfying one of the following conditions: 
$\mu$ is nonatomic and $\mu(\Omega)=\infty$,

$\mu$ is nonatomic and $\mu(\Omega)<\infty$,

$\mu$ is completely atomic, all atoms having equal measure

1 , and $\mu(\Omega)=\infty$.

$\mathscr{C}(\Omega), \mathscr{P}(\Omega)$ denote respectively the measurable and nonnegative measurable functions on $\Omega$. A function norm is a mapping $\rho: \mathscr{P}(\Omega) \rightarrow$ $[0, \infty]$ which, for all $f, f_{n} \in \mathscr{P}(\Omega)$, all scalars $\lambda>0$, satisfies:

$$
\begin{gathered}
\rho(f)=0 \Leftrightarrow f=0 \quad \mu \text { - a.e. } ; \\
\rho(\lambda f)=\lambda \rho(f) ; \quad f_{1} \leqq f_{2} \quad \mu \text { - a.e. } \Rightarrow \rho\left(f_{1}\right) \leqq \rho\left(f_{2}\right) ; \\
\rho\left(f_{1}+f_{2}\right) \leqq \rho\left(f_{1}\right)+\rho\left(f_{2}\right) ; \\
\mu(E)<\infty \Rightarrow \rho\left(\chi_{E}\right)<\infty \quad\left({ }^{1}\right) ; \\
\mu(E)<\infty \Rightarrow \int_{E} f d \mu \leqq A_{E} \rho(f), \quad \text { for some } A_{E}<\infty ; \\
f_{n} \uparrow f \mu \text { - a.e. } \Rightarrow \rho\left(f_{n}\right) \uparrow \rho(f) \quad \text { (Fatou property). }
\end{gathered}
$$

The space $X=L^{\circ}$ consists of all functions $f \in \mathscr{L}(\Omega)$ for which $\rho(|f|)<\infty$. When functions differing on at most a null-set are identified, $X$ is a Banach space under the norm $\|f\|_{X}=\rho(|f|)$, called a Banach function space. If $X$ contains, along with a function $f_{1}$, every function $f_{2}$ equimeasurable with $f_{1}$, we say that $X$ is a rearrangement-invariant space; we may and shall assume that if $f_{1}$ and $f_{2}$ are equimeasurable then $\left\|f_{1}\right\|_{X}=\left\|f_{2}\right\|_{X}$ (cf. [7], §16).

If a space $X$ has all the properties of a rearrangement-invariant space except that instead of the Fatou property (2.9) it satisfies the weaker Riesz-Fischer property

$$
f_{n} \in X, \sum_{n=1}^{\infty} \rho\left(f_{n}\right)<\infty \Rightarrow \rho\left(\sum_{n=1}^{\infty} f_{n}\right)<\infty,
$$

we shall say that $X$ is a Riesz-Fischer space (cf. [8], Notes I and II).

The nonincreasing rearrangement $f^{*}$ of $f$ is defined as in [2], and the Hardy maximal rearrangement $f^{* *}$ is given by

$$
f^{* *}(t)=\frac{1}{t} \int_{0}^{t} f^{*}(s) d s, \quad 0<t<\infty .
$$

We shall make frequent use of the following well-known inequality (cf. [7], §10)

$$
\left(f_{1}+f_{2}\right)^{* *} \leqq f_{1}^{* *}+f_{2}^{* *}
$$

\footnotetext{
${ }^{1} \chi_{E}$ denotes the characteristic function of a set $E$.
} 
The domain of definition of $f^{*}, f \in \mathscr{C}(\Omega)$, will be denoted by $\Omega^{*}$; thus if $\Omega$ satisfies (2.1) (resp. (2.2), (2.3)) we set $\Omega^{*}=R^{+}$(resp. $\left.[0, \mu(\Omega)], Z^{+}\right)$. To each rearrangement-invariant space $X$ on $\Omega$ there corresponds (via the Luxemburg representation theorem [7], §12) a rearrangement-invariant space $X^{*}$ on $\Omega^{*}$. The norms on $X$ and $X^{*}$ are related by

$$
\|f\|_{X}=\left\|f^{*}\right\|_{X^{*}},
$$

$f \in X$

When $X=L^{\rho}$ we shall write $X^{*}=L^{\rho^{*}}$.

The associate space $X^{\prime}$ of $X$ is defined as in [2]; note the resulting Hölder inequality (setting $a=\mu(\Omega)$ ):

$$
\int_{\Omega}|f g| d \mu \leqq \int_{0}^{a} f^{*}(t) g^{*}(t) d t \leqq\|f\|_{X}\|g\|_{X^{\prime}}, \quad f \in X, g \in X^{\prime} .
$$

The dilation operators $E_{s}, F_{s}, 0<s<\infty$, and $G_{s}, s$ or $s^{-1} \in Z^{+}, 2$ are defined as follows:

When $\Omega^{*}=R^{+}, 0<s<\infty$, set

$$
\left(E_{s} f\right)(t)=f(s t), \quad 0<t<\infty, f \in \mathscr{M}\left(R^{+}\right) .
$$

When $\Omega^{*}=[0, a]$ and $0<s<\infty$, set

$$
\left(F_{s} f\right)(t)=f(s t), \quad 0 \leqq t \leqq a, \quad f \in \mathscr{M}([0, a]),
$$

where $f(t)$ is defined to have value 0 if $t>a$.

When $\Omega^{*}=Z^{+}$and $m \in Z^{+}$, set

$$
\begin{aligned}
\left(G_{m} f\right)(n) & =f(m n), \\
\left(G_{m^{-1}} f\right)(n) & =f([(n-1) / m]+1) \quad n \in Z^{+}, f \in \mathscr{M}\left(Z^{+}\right),
\end{aligned}
$$

where $[\alpha]$ denotes the integer part of $\alpha$.

Now let $X$ be a rearrangement-invariant space on $\Omega$. If $\Omega^{*}=R^{+}$ we define $\left\|E_{s}\right\|_{(X)}$ by

$$
\left\|E_{s}\right\|_{(X)}=\sup \left\{\left\|E_{s} f^{*}\right\|_{X^{*}}:\|f\|_{X} \leqq 1\right\}, \quad 0<s<\infty ;
$$

in case $\Omega^{*}=[0, a], Z^{+}$there are analogous formulas for $\left\|F_{s}\right\|_{(X)}$ and $\left\|G_{s}\right\|_{(X)}$, respectively.

The Boyd indices ([2]) of $X$ are defined in the nonatomic case by

$$
\alpha(X)=\lim _{s \rightarrow 0} \frac{-\log \left\|E_{s}\right\|_{(X)}}{\log s} ; \quad \beta(X)=\lim _{s \rightarrow \infty} \frac{-\log \left\|E_{s}\right\|_{(X)}}{\log s}
$$

(with $E_{s}$ replaced by $F_{s}$ if $\Omega^{*}=[0, a]$ ), and in the atomic case by

$$
\alpha(X)=\lim _{m \rightarrow \infty} \frac{\log \left\|G_{m^{-1}}\right\|_{(X)}}{\log m} ; \quad \beta(X)=\lim _{m \rightarrow \infty} \frac{-\log \left\|G_{m}\right\|_{(X)}}{\log m} .
$$

2 The notation used here differs from that of Boyd ([2]). 
THEOREM 2.1 (Boyd [2]). Let $X$ be a rearrangement-invariant space on $(\Omega, \mathscr{T}, \mu)$. Then the limits (2.18) (or (2.19) as appropriate) exist and

$$
0 \leqq \beta(X) \leqq \alpha(X) \leqq 1
$$

Moreover, if $X^{\prime}$ is the associate space of $X$, then

$$
\alpha\left(X^{\prime}\right)=1-\beta(X) ; \quad \beta\left(X^{\prime}\right)=1-\alpha(X) .
$$

When $X$ is $L^{p}$ or a Lorentz space $L^{p r}$ both indices are equal to $p^{-1}$.

3. The Calderón maximal operator $S$. For each $f \in L^{1}\left(R^{+}\right)$, set

$$
(S f)(t)=\int_{0}^{1 / t} f(s) d s+\frac{1}{2} t^{-1 / 2} \int_{1 / t}^{\infty} s^{-1 / 2} f(s) d s, \quad 0<t<\infty .
$$

The operator $S$ so defined is precisely the Calderón maximal operator $S=S(\sigma)$ ([3], p. 288) for the segment $\sigma$ in the plane joining the points $(1,0)$ and $(1 / 2,1 / 2)$. It is a simple matter to check that $S\left(f^{*}\right)$ is nonnegative, nonincreasing and continuous on $R^{+}([3], \mathrm{p} .288)$ and hence that $\left(S\left(f^{*}\right)\right)^{*}=S\left(f^{*}\right)$. In subsequent sections we shall need to consider the maximal average (cf. (2.11)) $\left(S f^{*}\right)^{* *}$ of $S f^{*}$.

LeMma 3.1. For any $f \in L^{1}\left(R^{+}\right)$,

$$
\left(S f^{*}\right)^{* *}(t)=\int_{0}^{1 / t} f^{*}(s) d s+t^{-1 / 2} \int_{1 / t}^{\infty} s^{-1 / 2} f^{*}(s) d s, \quad 0<t<\infty .
$$

Furthermore, we have,

$$
S\left(f_{1}^{*}+f_{2}^{*}\right)(t)=\left(S f_{1}^{*}\right)^{* *}(t)+\left(S f_{2}^{*}\right)^{* *}(t), \quad 0<t<\infty,
$$

and, if $f_{1}, f_{2} \in L^{1}(T)$,

$$
S\left(\left(f_{1}+f_{2}\right)^{*}\right)^{* *}(t) \leqq\left(S f_{1}^{*}\right)^{* *}(t)+\left(S f_{2}^{*}\right)^{* *}(t), \quad 0<t<\infty .
$$

Proof. Equation (3.2) is established by a routine change in the order of integration. Note the similarity to (3.1). Putting $f=f_{1}^{*}+$ $f_{2}^{*}$, we have $f^{*}=\left(f_{1}^{*}+f_{2}^{*}\right)^{*}=f_{1}^{*}+f_{2}^{*}=f$, and (3.3) follows directly from (3.2).

If $f \in L^{1}(T)$, then $f^{*}$ is supported in $[0,1]$ and so from (3.2) we see that $\left(S f^{*}\right)^{* *}$ has the constant value $\int_{0}^{1} f^{*}(s) d s$ on $[0,1]$. Hence, if $f_{1}, f_{2} \in L^{1}(T)$ we have for all $t, 0<t \leqq 1$,

$$
\begin{aligned}
S\left(\left(f_{1}+f_{2}\right)^{*}\right)^{* *}(t) & =\left\|f_{1}+f_{2}\right\|_{L^{1}(T)} \leqq\left\|f_{1}\right\|_{L^{1}(T)}+\left\|f_{2}\right\|_{L^{1}(T)} \\
& =\left(S f_{1}^{*}\right)^{* *}(t)+\left(S f_{2}^{*}\right)^{* *}(t) .
\end{aligned}
$$


Thus, it remains only to show that the preceding inequality persists for all $t>1$.

Now, if $t>1$, we set

$$
\psi_{t}(s)=1, \quad 0 \leqq s \leqq 1 / t ; \quad \psi_{t}(s)=(s t)^{-1 / 2}, \quad 1 / t \leqq s \leqq 1,
$$

so that by (3.2) we have

$$
\left(S f^{*}\right)^{* *}(t)=\int_{0}^{1} f^{*}(s) \psi_{t}(s) d s,
$$

Note that $\psi_{t}$ is continuous and nonincreasing on [0,1]. If $f_{1}, f_{2} \in L^{1}(T)$, we deduce from (2.12) that

$$
\int_{0}^{u}\left(f_{1}+f_{2}\right)^{*}(s) d s \leqq \int_{0}^{u}\left(f_{1}^{*}+f_{2}^{*}\right)(s) d s, \quad 0<u \leqq 1,
$$

and so it follows by a theorem of Hardy (cf. [7], §5) that

$$
\int_{0}^{1}\left(f_{1}+f_{2}\right)^{*}(s) \psi_{t}(s) d s \leqq \int_{0}^{1}\left(f_{1}^{*}(s) \psi_{t}(s)+f_{2}^{*}(s) \psi_{t}(s)\right) d s
$$

i.e.,

$$
S\left(\left(f_{1}+f_{2}\right)^{*}\right)^{* *}(t) \leqq\left(S f_{1}^{*}\right)^{* *}(t)+\left(S f_{2}^{*}\right)^{* *}(t), \quad t>1 .
$$

This completes the proof.

It follows easily from (3.2) that $\left(S f^{*}\right)^{* *}$ is nonnegative, nonincreasing and continuous on $R^{+}$.

LEMma 3.2 (Calderón [3], p. 288). The operator $S$ is of (strong) type $(1, \infty)$ and of weak type $(2,2)$. The same is therefore true of the operator $f \rightarrow\left(S f^{*}\right)^{* *}$.

The next theorem justifies the terminology 'maximal operator' which we have applied to $S$.

THEOREM 3.3 (Calderón [3], p. 290). Let $U$ be any linear operator defined on $L^{1}(T)$ whose values are functions defined on $Z$. If $U$ is of type $(1, \infty)$ and weak type $(2,2)$ then

$$
(U f)^{*} \leqq c S f^{*}
$$

where $c$ is a constant independent of $f$.

Since the Fourier transform $\mathscr{T}$ is of strong (hence weak) types $(1, \infty)$ and $(2,2)$ we deduce the following result:

Corollary 3.4. For each $f \in L^{1}(T)$, there is the estimate 


$$
(\mathscr{T} f)^{*} \leqq c S f^{*},
$$

where $c$ is a constant independent of $f$.

4. The space $\hat{X}_{0}$. Let $X$ be an arbitrary rearrangement-invariant space on the circle $T$ (no restrictions on the indices are necessary at this stage). In this case the conditions (2.7) and (2.8) imply

$$
L^{\infty} \cong X \subseteq L^{1}
$$

with continuous embeddings, i.e., there are constants $c_{i}=c_{i}(X), i=1,2$, such that

$$
\|f\|_{1} \leqq c_{1}\|f\|_{X}, f \in X ;\|f\|_{X} \leqq c_{2}\|f\|_{\infty}, \quad f \in L^{\infty} .
$$

The set $\hat{X}_{0}$ of functions on $Z$ is defined by

$$
\hat{X}_{0}=\left\{g=(g(n))_{n=-\infty}^{\infty}: g^{* *} \leqq\left(S f^{*}\right)^{* *} \text { for some } f \in X\right\}
$$

and we set

$$
\|g\| \hat{x}_{0}=\inf \left\{\|f\|_{x}: g^{* *} \leqq\left(S f^{*}\right)^{* *}\right\} ; \quad g \in \hat{X}_{0} ;
$$

equivalently, by (2.13),

$$
\|g\| \hat{X}_{0}=\inf \left\{\left\|f^{*}\right\|_{X^{*}}: g^{* *} \leqq\left(S f^{*}\right)^{* *}\right\}, \quad g \in \hat{X}_{0} .
$$

It will be established in the following series of lemmas that the space $\hat{X}_{0}$ so defined is a Riesz-Fischer space which fails in general to satisfy the Fatou property (2.9) (cf. §7). However, we show in the next section how to construct from $\hat{X}_{0}$ a second space $\hat{X}$ with all of the desired properties.

For notational convenience we shall use $\|(\cdot)\|$ to denote the norm on $X$ and $\|(\cdot)\|_{0}$ to denote the norm on $\hat{X}_{0}$.

LEMMA 4.1. If $g \in \hat{X}_{0}$ then

$$
\|g\|_{\infty} \leqq c\|g\|_{0}
$$

where $c$ is a constant independent of $g$.

Proof. Since $g \in \hat{X}_{0}$, there exists a function $f \in X$ satisfying

$$
g^{* *} \leqq\left(S f^{*}\right)^{* *} \text {. }
$$

But then by Lemma 3.2 (or directly)

$$
\|g\|_{\infty}=\left\|g^{* *}\right\|_{L^{\infty}\left(R^{+}\right)} \leqq\left\|\left(S f^{*}\right)^{* *}\right\|_{L^{\infty}\left(R^{+}\right)} \leqq\left\|f^{*}\right\|_{L^{1}\left(R^{+}\right)}=\|f\|_{L^{1}(T)} .
$$

Combining this last estimate with (4.2) we have $\|g\|_{\infty} \leqq c\|f\|$ and so 
taking the infimum of the right-hand side over all $f$ satisfying (4.6), we deduce from (4.4) that $\|g\|_{\infty} \leqq c\|g\|_{0}$.

LEMma 4.2. $\|g\|_{0}=0 \Leftrightarrow g=0$.

Proof. If $g=0$ then, by (4.4), $\|g\|_{0}=0$. The reverse implication is a direct consequence of Lemma 4.1 .

The proof of the next lemma is obvious and we omit it.

Lemma 4.3. (a) $\|\lambda g\|_{0}=|\lambda|\|g\|_{0}$,

(b) $\left|g_{1}\right| \leqq\left|g_{2}\right| \Longrightarrow\left\|g_{1}\right\|_{0} \leqq\left\|g_{2}\right\|_{0}$.

LEMma 4.4. $\left\|g_{1}+g_{2}\right\|_{0} \leqq\left\|g_{1}\right\|_{0}+\left\|g_{2}\right\|_{0}$.

Proof. Let $g_{1}, g_{2} \in \hat{X}_{0}$ and fix $\varepsilon>0$. Then there exist $f_{1}, f_{2} \in X$ with $g_{i}^{* *} \leqq\left(S f_{i}^{*}\right)^{* *}$ and $\left\|f_{i}\right\|<\left\|g_{i}\right\|_{0}+\varepsilon / 2, i=1,2$. It follows from (2.12) and (3.3) that

$$
\begin{aligned}
\left(g_{1}+g_{2}\right)^{* *} & \leqq g_{1}^{* *}+g_{2}^{* *} \leqq\left(S f_{1}^{*}\right)^{* *}+\left(S f_{2}^{*}\right)^{* *} \\
& =S\left(f_{1}^{*}+f_{2}^{*}\right)^{* *}=S\left(\left(f_{1}^{*}+f_{2}^{*}\right)^{*}\right)^{* *} .
\end{aligned}
$$

Hence, from (4.5) and (2.13)

$$
\left\|g_{1}+g_{2}\right\|_{0} \leqq\left\|f_{1}^{*}+f_{2}^{*}\right\|_{X^{*}} \leqq\left\|f_{1}^{*}\right\|_{X^{*}}+\left\|f_{2}^{*}\right\|_{X^{*}}=\left\|f_{1}\right\|+\left\|f_{2}\right\|
$$

and by the choice of $f_{1}, f_{2}$

$$
\left\|g_{1}+g_{2}\right\|_{0} \leqq\left\|g_{1}\right\|_{0}+\left\|g_{2}\right\|_{0}+\varepsilon \text {. }
$$

Since $\varepsilon$ is arbitrary, this completes the proof. $\hat{X}_{0}$.

Lemma 4.5. Characteristic functions of all finite sets belong to

Proof. If $g$ is the characteristic function of a set of $n$ points, then $g^{* *}(t)=1,0<t<n ; g^{* *}(t)=n / t, t \geqq n$. Now the constant function $f(\theta)=n, 0<\theta<2 \pi$, belongs to $L^{\infty}(T)$, hence, by (4.1), to $X$. It is a simple matter to check that $g^{* *} \leqq\left(S f^{*}\right)^{* *}$ and hence that $g \in \hat{X}_{0}$. We omit the details.

Lemma 4.6. For each $N \in Z^{+}$there is a constant $A_{N}<\infty$ such that

$$
\sum_{|n| \leqq N}|g(n)| \leqq A_{N}\|g\|_{0}, \quad g \in \hat{X}_{0}
$$

Proof. Since $\sum_{|n| \leqq N}|g(n)| \leqq(2 N+1)\|g\|_{\infty}$, the estimate (4.8) 
follows directly from (4.6).

Lemma 4.7. $\hat{X}_{0}$ has the Riesz-Fischer property.

Proof. Let $\left(g_{n}\right)_{n=1}^{\infty}$ be a sequence of functions $g_{n} \in \hat{X}_{0}$ with $\sum_{n=1}^{\infty}\left\|g_{n}\right\|_{0}<\infty$. By (2.10), we must show that $g=\sum_{n=1}^{\infty} g_{n}$ belongs to $\hat{X}_{0}$. Now for each $n \in Z^{+}$there exists a function $f_{n} \in X$ such that

$$
g_{n}^{* *} \leqq\left(S f_{n}^{*}\right)^{* *}, \quad\left\|f_{n}\right\| \leqq\left\|g_{n}\right\|_{0}+2^{-n} .
$$

It follows that

$$
\sum_{n=1}^{\infty}\left\|f_{n}^{*}\right\|_{X^{*}}=\sum_{n=1}^{\infty}\left\|f_{n}\right\| \leqq \sum_{n=1}^{\infty}\left\|g_{n}\right\|_{0}+\sum_{n=1}^{\infty} 2^{-n}<\infty .
$$

But $X^{*}$ has the Riesz-Fischer property so from (4.9) we deduce that the function $f=f^{*}=\sum_{n=1}^{\infty} f_{n}^{*}$ belongs to $X^{*}$.

Again using (4.9) and (4.2) we see that for each fixed $t \in R^{+}$,

$$
\sum_{n=1}^{\infty}\left(S f_{n}^{*}\right)^{* *}(t) \leqq \sum_{n=1}^{\infty}\left\|f_{n}^{*}\right\|_{L^{1}\left(R^{+}\right)} \leqq c \sum_{n=1}^{\infty}\left\|f_{n}^{*}\right\|_{X^{*}}<\infty .
$$

Hence by the dominated convergence theorem and (3.2)

$$
\begin{aligned}
\sum_{n=1}^{\infty}\left(S f_{n}^{*}\right)^{* *}(t) & =\sum_{n=1}^{\infty}\left(\int_{0}^{1 / t} f_{n}^{*}(s) d s+t^{-1 / 2} \int_{1 / t}^{\infty} s^{-1 / 2} f_{n}^{*}(s) d s\right) \\
& =\int_{0}^{1 / t} \sum_{n=1}^{\infty} f_{n}^{*}(s) d s+t^{-1 / 2} \int_{1 / t}^{\infty} s^{-1 / 2} \sum_{n=1}^{\infty} f_{n}^{*}(s) d s \\
& =\left(S f^{*}\right)^{* *}(t) .
\end{aligned}
$$

But then

$$
g^{* *}=\left(\sum_{n=1}^{\infty} g_{n}\right)^{* *} \leqq \sum_{n=1}^{\infty} g_{n}^{* *} \leqq \sum_{n=1}^{\infty}\left(S f_{n}^{*}\right)^{* *}=\left(S f^{*}\right)^{* *} .
$$

Since $f^{*} \in X^{*}$, it follows from (4.5) that $g \in \hat{X}_{0}$. This completes the proof.

Theorem 4.8. Let $X$ be any rearrangement-invariant space on T. Then the space $\hat{X}_{0}$ is a Riesz-Fischer space and $\mathscr{T}$ is a bounded linear operator from $X$ into $\hat{X}_{0}$.

Proof. That $\hat{X}_{0}$ is a Riesz-Fischer space is the content of Lemmas $4.2, \cdots, 4.7$. If $f \in X$ then by $(3.4),(\mathscr{T} f)^{*} \leqq c S f^{*}=S\left(c f^{*}\right)$ and so $(\mathscr{T} f)^{* *} \leqq\left(S\left(c f^{*}\right)\right)^{* *}$. Since $c f \in X$ it follows from (4.4) that $\mathscr{T} f \in \hat{X}_{0}$ and that $\|\mathscr{T} f\|_{0} \leqq\|c f\|_{X}=c\|f\|_{X}$. This completes the proof.

5. The space $\hat{X}$. We denote by $\chi_{n}$ the characteristic function 
of the set $\{-n, \cdots,-1,0,1, \cdots, n\} \subseteq Z$, and for each function $g$ defined on $Z$ we set

$$
\|g\|_{\hat{X}}=\sup _{n}\left\|g \chi_{n}\right\|_{0}=\lim _{n \rightarrow \infty}\left\|g \chi_{n}\right\|_{0} \text {. }
$$

The space $\hat{X}$ then consists of all functions $g$ for which $\|g\|_{\hat{X}}<\infty$.

Note that if $g \in \hat{X}_{0}$ then $\left|g \chi_{n}\right| \leqq|g|$ so by Lemma 4.3 (b), $\left\|g \chi_{n}\right\|_{0} \leqq$ $\|g\|_{0}$. From (5.1) we deduce that $g \in \hat{X}$. Thus

$$
\hat{X}_{0} \sqsubseteq \hat{X}
$$

and

$$
\|g\|_{\hat{X}} \leqq\|g\|_{0}, \quad g \in \hat{X}_{0} \text {. }
$$

The properties $(2.4), \cdots,(2.8)$ for $\hat{X}$ are easily verified from the corresponding properties for $\hat{X}_{0}$ (Lemmas $\left.4.2, \cdots, 4.6\right)$. To see that $\hat{X}$ is rearrangement-invariant, let $g_{1} \in \hat{X}$ and suppose that $g_{1}$ and $g_{2}$ are equimeasurable. If $N \in Z^{+}$, then $g_{2} \chi_{N}$ assumes only finitely many values. But then the fact that $g_{1}$ and $g_{2}$ are equimeasurable implies the existence of $M \in Z^{+}$such that the values of $g_{2} \chi_{N}$ all are assumed by $g_{1} \chi_{M}$. Hence $\left(g_{2} \chi_{N}\right)^{*} \leqq\left(g_{1} \chi_{M}\right)^{*}$ so by Lemma $4.3(\mathrm{~b}),\left\|g_{2} \chi_{N}\right\|_{0} \leqq$ $\left\|g_{1} \chi_{M}\right\|_{0} \leqq\left\|g_{1}\right\|_{\hat{X}}$. This holds for all $N \in Z^{+}$so we deduce from (5.1) that $g_{2} \in \hat{X}$ and $\left\|g_{2}\right\|_{\hat{X}} \leqq\left\|g_{1}\right\|_{\hat{X}}$. Interchanging the roles of $g_{1}$ and $g_{2}$ we obtain the reverse inequality and so finally we have $\left\|g_{1}\right\| \hat{x}=\left\|g_{2}\right\| \hat{x}$.

The Fatou property is "built-in" to the norm on $\hat{X}$. Indeed it is clear from (5.1) that $\left\|g \chi_{n}\right\|_{\hat{X}} \uparrow\|g\|_{\hat{x}}$ as $n \rightarrow \infty$, for any $g \in \hat{X}$, and by Theorem 5.9 of ([8], Note II,) this is enough to ensure that $\hat{X}$ has the Fatou property. The next theorem is useful in identifying the space $\hat{X}$ when $X$ is given in concrete terms (e.g. a Lorentz or Lebesgue space); see $\S 7$. We omit the obvious proof.

THEOREM 5.1. Let $X$ be a rearrangement-invariant space on $T$. Then $\hat{X}=\hat{X}_{0}$ (with identical norms) if and only if $\hat{X}_{0}$ has the Fatou property.

THEOREM 5.2. Let $X$ be a rearrangement-invariant space on $T$. Then the space $\hat{X}$ is a rearrangement-invariant space on $Z$ and $\mathscr{T}$ is a bounded linear operator from $X$ into $\hat{X}$.

Proof. That $\hat{X}$ is a rearrangement-invariant space has been established above. The boundedness of $\mathscr{T}$ follows from (5.2), (5.3), and Theorem 4.8.

6. Indices of $\hat{X}$. The first theorem in this section enables us to estimate the indices of $\hat{X}$ in terms of those of $\hat{X}_{0}$. 
THEOREM 6.1. Let $X$ be any rearrangement-invariant space on T. Then the indices of $\hat{X}_{0}$ and $\hat{X}$ are related $b y$

$$
\beta\left(\hat{X}_{0}\right) \leqq \beta(\hat{X}) \leqq \alpha(\hat{X}) \leqq \alpha\left(\hat{X}_{0}\right)
$$

Proof. Fix $g \in \hat{X}$ and $m \in Z^{+}$. It is clear that $\left(G_{m} g^{*}\right) \chi_{n}=G_{m}\left(g^{*} \chi_{m n}\right)$ for all $n \in Z^{+}$, and so

$$
\begin{aligned}
\left\|G_{m} g^{*}\right\|_{\hat{x}} & =\sup _{n}\left\|\left(G_{m} g^{*}\right) \chi_{n}\right\|_{0}=\sup _{n}\left\|G_{m}\left(g^{*} \chi_{m n}\right)\right\|_{0} \\
& \leqq\left\|G_{m}\right\|\left(\hat{x}_{0}\right) \sup _{n}\left\|g^{*} \chi_{m n}\right\|_{0}=\left\|G_{m}\right\|_{\left(\hat{x}_{0}\right)}\|g\|_{\hat{x}} .
\end{aligned}
$$

It follows from (2.18) that $\left\|G_{m}\right\|_{(\hat{X})} \leqq\left\|G_{m}\right\|_{\left(\hat{X}_{0}\right)}$ and hence by (2.20) that $\beta\left(\hat{X}_{0}\right) \leqq \beta(\hat{X})$. An entirely analogous proof for the operators $G_{1 / m}$ now shows that $\left\|G_{1 / m}\right\|_{(\hat{X})} \leqq\left\|G_{1 / m}\right\|_{\left(\hat{X}_{0}\right)}$ and hence that $\alpha(\hat{X}) \leqq \alpha\left(\hat{X}_{0}\right)$. The proof is completed by applying (2.21). lemma.

In order to compute the indices of $\hat{X}_{0}$ we need the following

LEMMA 6.2. Let $f^{*} \in L^{1}\left(R^{+}\right), g^{*} \in l^{\infty}\left(Z^{+}\right)$and fix $m \in Z^{+}$. If $g^{* *} \leqq\left(S f^{*}\right)^{* *}$ then the following inequalities also are valid:

$$
\begin{gathered}
\left(G_{1 / m} g^{*}\right)^{* *} \leqq m S\left(E_{m} f^{*}\right)^{* *} \\
\left(G_{m} g^{*}\right)^{* *} \leqq m^{-1} S\left(E_{1 / m} f^{*}\right)^{* *} .
\end{gathered}
$$

Proof. We show first that

$$
\left(G_{1 / m} g^{*}\right)^{* *}=E_{1 / m}\left(g^{* *}\right) \text {. }
$$

Indeed, if $t \in R^{+}$, then $t$ has a unique decomposition $t=k m+\alpha$, $k \in Z^{+}, 0 \leqq \alpha<m$, so from $(2.17)$

$$
\begin{aligned}
\left(G_{1 / m} g^{*}\right)^{* *}(t) & =\frac{1}{t} \int_{0}^{t}\left(G_{1 / m} g^{*}\right)(s) d s \\
& =\frac{1}{t}\left[\int_{0}^{m} g^{*}(1) d s+\cdots+\int_{(k-1) m}^{k m} g^{*}(k) d s+\int_{k m}^{t} g^{*}(k+1) d s\right] \\
& =\frac{m}{t}\left[\sum_{j=1}^{k} g^{*}(j)+\frac{\alpha}{m} g^{*}(k+1)\right]=\frac{m}{t} \int_{0}^{t / m} g^{*}(s) d s \\
& =g^{* *}(t / m)=E_{1 / m}\left(g^{* *}\right)(t) .
\end{aligned}
$$

The identity

$$
E_{1 / m}\left[\left(S f^{*}\right)^{* *}\right]=m S\left(E_{m} f^{*}\right)^{* *}
$$

is established by a similar "change of variable" argument. The desired 
result (6.2) now follows from (6.4), the hypothesis $g^{* *} \leqq\left(S f^{*}\right)^{* *}$, and (6.5). The inequality (6.3) can be established in similar fashion.

THEOREM 6.3. Let $X$ be any rearrangement-invariant space on T. Then

$$
\alpha\left(\hat{X}_{0}\right) \leqq 1-\beta(X)
$$

Proof. Fix $m \in Z^{+}$and let $\delta$ be any number satisfying $\delta>1$. Then for each $g \in \hat{X}_{0}, g \neq 0$, there exists a function $f \in X$ such that $g^{* *} \leqq\left(S f^{*}\right)^{* *}$ and $\|f\|<\delta\|g\|_{0}$. It follows from (6.2) that $\left(G_{1 / m} g^{*}\right)^{* *} \leqq$ $m S\left(F_{m} f^{*}\right)^{* *}$, since $E_{s}=F_{s}$ for $s>1$, and so from (4.5) we deduce that $G_{1 / m} g^{*} \in \hat{X}_{0}$ and

$$
\left\|G_{1 / m} g^{*}\right\|_{0} \leqq m\left\|F_{m} f^{*}\right\|_{X^{*}} \leqq m\left\|F_{m}\right\|_{(x)}\|f\| \cdot
$$

Hence, by choice of $f$, we have

$$
\left\|G_{1 / m} g^{*}\right\|_{0} \leqq m \delta\left\|F_{m}\right\|_{(X)}\|g\|_{0} \text {. }
$$

This last estimate holds for all $g \in \hat{X}_{0}$ so we find that $\left\|G_{1 / m}\right\|_{\left(\hat{X}_{0}\right)} \leqq$ $m \delta\left\|F_{m}\right\|_{(x)}$. This in turn holds for all $\delta>1$ so we have $\left\|G_{1 / m}\right\|_{\left(\hat{X}_{0}\right)} \leqq$ $m\left\|F_{m}\right\|_{(X)}$, and by (2.19) and (2.20) this suffices to show that $\alpha\left(\hat{X}_{0}\right) \leqq$ $1-\beta(X)$. The proof is complete.

The analogue of (6.6) for the lower index $\beta\left(\hat{X}_{0}\right)$ is a little more difficult to establish, although the proof follows the same lines as that of Theorem 6.3. The difficulty arises from the fact that we cannot, in general replace $E_{1 / m} f^{*}$ by $F_{1 / m} f^{*}$ (since the former is supported in $[0, m]$, the latter in $[0,1])$. However, it is fairly straightforward to estimate their difference and for this we need the following lemma. For each $m \in Z^{+}$, we set

$$
f_{m}(t)=t^{-1 / 2} \chi_{[2-m, 1]}(t), \quad 0 \leqq t \leqq 1 .
$$

LEMMA 6.4. Let $Y$ be a rearrangement-invariant space on $[0,1]$ with lower index $\beta$ satisfying $0 \leqq \beta \leqq 1 / 2$. Then for each $\varepsilon>0$, there is a constant $c=c(\varepsilon)$, depending only upon $\varepsilon$ (and $Y$ ), such that

$$
\left\|f_{m}\right\|_{Y} \leqq c(\varepsilon)\left(2^{m}\right)^{1 / 2-\beta+\varepsilon}, \quad m \in Z^{+} \text {. }
$$

Proof. It is clear from $(6.7)$ that $f_{m}^{*}(t)=\left(t+2^{-m}\right)^{-1 / 2} \chi_{\left(0,1-2^{-m}\right]^{(t)}}$, $0 \leqq t \leqq 1$. Thus, if $f_{m k}$ is defined by

$$
f_{m k}=f_{m}^{*} \chi_{\left(2^{k-m-1}-2^{-m}, 2^{\left.k-m_{-2}-m\right]}\right.}, \quad k=1,2, \cdots, m,
$$

we have $f_{m}^{*}=\sum_{k=1}^{m} f_{m k}$ and hence

$$
\left\|f_{m}\right\|_{Y}=\left\|f_{m}^{*}\right\|_{Y} \leqq \sum_{k=1}^{m}\left\|f_{m k}\right\|_{Y}=\sum_{k=1}^{m}\left\|f_{m k}^{*}\right\|_{Y} \cdot
$$


Now if $h(t)=(t+1)^{-1 / 2}, 0 \leqq t \leqq 1$, we have

$$
f_{m k}^{*}(t)=\left(t+2^{-m+k-1}\right)^{-1 / 2} \chi_{\left(0,2^{-m+k-1}\right]}(t)=\left(2^{m-k+1}\right)^{1 / 2}\left(F_{2^{m-k+1}}(h)\right)(t)
$$

so from (6.9) we deduce that

$$
\left\|f_{m}\right\|_{Y} \leqq \sum_{k=1}^{m}\left(2^{2 n-k+1}\right)^{1 / 2}\left\|F_{2^{m-k+1}}\right\|_{(Y)}\|h\|_{Y}=c_{0} \sum_{k=1}^{m} 2^{k / 2}\left\|F_{2^{k}}\right\|_{(Y)} .
$$

It follows from (2.19) that there is a positive integer $M=M(\varepsilon)$ such that $k \geqq M$ implies $\left\|F_{2^{k}}\right\|_{(Y)} \leqq\left(2^{k}\right)^{-\beta+\varepsilon}$. Hence, if $m \geqq M$, we have from $(6.10)$

$$
\begin{aligned}
\left\|f_{m}\right\|_{(Y)} & \leqq c_{0} \sum_{k=1}^{M} 2^{k / 2}\left\|F_{2^{k}}\right\|_{(Y)}+c_{0} \sum_{k=M H+1}^{m} 2^{k / 2}\left(2^{k}\right)^{-\beta+\varepsilon} \\
& \leqq c_{0} \sum_{k=1}^{M} 2^{k / 2}+c_{0} \sum_{k=1}^{m}\left(2^{1 / 2-\beta+\varepsilon}\right)^{k},
\end{aligned}
$$

since $\left\|F_{s}\right\|_{(Y)} \leqq 1$ if $s \geqq 1$. The first term on the right-hand side is a constant, say $c_{1}$, depending only on $M$ and hence ultimately only upon $\varepsilon$; by hypothesis on $\beta$ we have $1 / 2-\beta+\varepsilon>0$, so the second term is dominated by a multiple of $\left(2^{1 / 2-\beta+\varepsilon}\right)^{m}$. Hence

$$
\left\|f_{m}\right\|_{Y} \leqq c_{1}(\varepsilon)+c_{2}(\varepsilon)\left(2^{m}\right)^{1 / 2-\beta+\varepsilon} \leqq c(\varepsilon)\left(2^{m}\right)^{1 / 2-\beta+\varepsilon}, \quad m \geqq M(\varepsilon),
$$

where $c=\max \left(c_{1}, c_{2}\right)$, and it is clear that by a suitable choice of constant $c(\varepsilon)$ this inequality can be made to persist for all $m \in Z^{+}$. This completes the proof.

THEOREM 6.5. Let $X$ be a rearrangement-invariant space on $T$ with upper index $\alpha=\alpha(X)$ satisfying $1 / 2 \leqq \alpha \leqq 1$. Then

$$
\beta\left(\hat{X}_{0}\right) \geqq 1-\alpha(X) \text {. }
$$

Proof. Fix $\varepsilon>0$ and $m \in Z^{+}$. If $g \in \hat{X}_{0}$ then there is a function $f \in X$ satisfying

$$
g^{* *} \leqq\left(S f^{*}\right)^{* *} .
$$

From the inequality (6.3) of Lemma 6.2 we have

$$
\left(G_{2^{m}} g^{*}\right)^{* *}(t) \leqq 2^{-m} S\left(E_{2^{-m}} f^{*}\right)^{* *}(t), \quad 0<t<\infty .
$$

Now it is routine to verify that for all $t \geqq 1$,

$$
\begin{aligned}
2^{-m} S\left(E_{2^{-m}} f^{* * *}\right)^{* *}(t)= & 2^{-m} S\left(F_{2^{-m}} f^{*}\right)^{* *}(t) \\
& +t^{-1 / 2}\left(2^{-m / 2} \int_{2^{-m}}^{1} s^{-1 / 2} f^{*}(s) d s\right),
\end{aligned}
$$

and hence by $(6.13)$ 


$$
\begin{aligned}
\left(G_{2^{m}} g^{*}\right)^{* *}(t) \leqq & 2^{-m} S\left(F_{2^{-m}} f^{*}\right)^{* *}(t) \\
& +t^{-1 / 2}\left(2^{-m / 2} \int_{2^{-m}}^{1} s^{-1 / 2} f^{*}(s) d s\right), \quad t \geqq 1 .
\end{aligned}
$$

In order to find a similar estimate for $t<1$, we observe that $\left(G_{2^{m}} g^{*}\right)^{* *}$ is constant on $[0,1]$ so for each $s, 0<s \leqq 1$, we have from (6.14) (with $t=1$ ),

$$
\begin{aligned}
\left(G_{2^{m}} g^{*}\right)^{* *}(s) & =\left(G_{2^{m}} g^{*}\right)^{* *}(1) \\
& \leqq 2^{-m} S\left(F_{2^{-m}} f^{*}\right)^{* *}(1)+\left(2^{-m / 2} \int_{2^{-m}}^{1} s^{-1 / 2} f^{*}(s) d s\right) \\
& \leqq 2^{-m} S\left(F_{2^{-m}} f^{*}\right)^{* *}(s)+\left(2^{-m / 2} \int_{2^{-m}}^{1} s^{-1 / 2} f^{*}(s) d s\right),
\end{aligned}
$$

the last inequality because $S\left((\cdot)^{*}\right)^{* *}$ decreases on $R^{+}$(cf. $\S 3$ ). Combining this with $(6.15)$ we have

$$
\begin{aligned}
\left(G_{2^{m}} g^{*}\right)^{* *}(t) \leqq & 2^{-m} S\left(F_{2^{-m}} f^{*}\right)^{* *}(t) \\
& +\varphi(t)\left(2^{-m / 2} \int_{2^{-m}}^{1} s^{-1 / 2} f^{*}(s) d s\right), \quad 0<t<\infty,
\end{aligned}
$$

where $\varphi(t)=\min \left(1, t^{-1 / 2}\right)$. Now if $h(t)=1,0 \leqq t \leqq 1$, it is a simple matter to check that $\varphi(t) \leqq\left(S h^{*}\right)^{* *}(t), 0<t<\infty$, so using (3.3) we can reduce (6.16) to the form

$$
\begin{aligned}
\left(G_{2^{m}} g^{*}\right)^{* *}(t) \leqq S\left(2^{-m} F_{2^{-m}} f^{*}+\left(2^{-m / 2} \int_{2^{-m}}^{1} s^{-1 / 2} f^{*}(s) d s\right) h^{*}\right)^{* *}(t) & , \\
& 0<t<\infty .
\end{aligned}
$$

It follows from (4.4) that $G_{2^{m}} g^{*} \in \hat{X}_{0}$ and

$$
\begin{aligned}
\left\|G_{2^{m}} g^{*}\right\|_{0} & \leqq 2^{-m}\left\|F_{2^{-m}} f^{*}\right\|_{X^{*}}+\left(2^{-m / 2} \int_{2^{-m}}^{1} s^{-1 / 2} f^{*}(s) d s\right)\left\|h^{*}\right\|_{X^{*}} \\
& \leqq 2^{-m}\left\|F_{2^{-m}}\right\|_{(X)}\|f\|+c_{0}\left(2^{-m / 2} \int_{2^{-m}}^{1} s^{-1 / 2} f^{*}(s) d s\right) .
\end{aligned}
$$

We estimate separately each of the terms on the right-hand side of (6.17). From (2.19) we note that there is a constant $M=M(\varepsilon)$ such that $m \geqq M$ implies $\left\|F_{2-m}\right\|_{(X)} \leqq\left(2^{m}\right)^{\alpha+\varepsilon}$. Hence, if $m \geqq M$,

$$
2^{-m}\left\|F_{2-m}\right\|_{(X)}\|f\| \leqq\left(2^{m}\right)^{\alpha-1+\varepsilon}\|f\| .
$$

To estimate the second term we invoke the Hölder inequality (2.14) to obtain

$$
\int_{2^{-m}}^{1} s^{-1 / 2} f^{*}(s) d s \leqq\left\|f^{*}\right\| X_{X^{*}}\left\|s^{-1 / 2} \chi_{\left(2^{-m, 1]}\right.}\right\|_{\left(X^{*}\right)} \cdot
$$

Now, by hypothesis, the upper index $\alpha$ of $X$ satisfies $1 / 2 \leqq \alpha \leqq 1$ so 
by (2.22) the lower index of $\left(X^{*}\right)^{\prime}$ is equal to $1-\alpha$ and hence lies between 0 and $1 / 2$. It follows from Lemma 6.4 and (6.19) that there is a constant $c=c(\varepsilon)$ such that

$$
2^{-m / 2} \int_{2^{-m}}^{1} s^{-1 / 2} f^{*}(s) d s \leqq c(\varepsilon)\|f\|_{X}\left(2^{m}\right)^{\alpha-1+\varepsilon}, \quad m \in Z^{+} .
$$

We can now combine (6.18) and (6.20) with (6.17) to obtain

$$
\left\|G_{2^{m}} g^{*}\right\|_{0} \leqq c_{1}(\varepsilon)\left(2^{m}\right)^{\alpha-1+\varepsilon}\|f\|, \quad m \geqq M(\varepsilon),
$$

and hence, taking the infimum over all $f$ satisfying (6.12),

$$
\left\|G_{2^{m}} g^{*}\right\|_{0} \leqq c_{1}(\varepsilon)\left(2^{m}\right)^{\alpha-1+\varepsilon}\|g\|_{0}, \quad m \geqq M(\varepsilon) \text {. }
$$

This is valid for all $g \in \hat{X}_{0}$ so (2.18) shows that

$$
\left\|G_{2^{m}}\right\|_{\left(\hat{X}_{0}\right)} \leqq c_{1}(\varepsilon)\left(2^{m}\right)^{\alpha-1+\varepsilon}, \quad m \geqq M(\varepsilon),
$$

and by (2.20) this in turn implies that $\beta\left(\hat{X}_{0}\right) \geqq 1-\alpha-\varepsilon$. Finally, since $\varepsilon$ is arbitrary, we have $\beta \geqq 1-\alpha$, and this completes the proof.

We are now in a position to prove our main result (Theorem A) which we restate as follows:

THeOREM 6.6. Let $X$ be a rearrangement-invariant space on $T$ with indices $\left(p^{-1}, p^{-1}\right), 1 \leqq p \leqq 2$. Then the space $\hat{X}$ is a rearrangement-invariant space on $Z$ with indices $\left(q^{-1}, q^{-1}\right), p^{-1}+q^{-1}=1$, and $\mathscr{T}$ is a bounded linear operator from $X$ into $\hat{X}$.

Proof. In view of Theorem 5.2 we need only show that the indices of $\hat{X}$ coincide and are equal to $q^{-1}$. But from $(6.1),(6.6)$, and $(6.11)$ we have

$$
q^{-1}=1-\alpha(X) \leqq \beta\left(\hat{X}_{0}\right) \leqq \beta(\hat{X}) \leqq \alpha(\hat{X}) \leqq \alpha\left(\hat{X}_{0}\right) \leqq 1-\beta(X)=q^{-1},
$$

and so the proof is complete.

When $2<p \leqq \infty$, it is no longer true that $\mathscr{T}$ maps $L^{p}$ into $l^{q}$. Indeed (cf. [9], p. 101), there are functions in $L^{\infty}$, hence in $L^{p}$, whose Fourier transforms do not lie in any of the classes $l^{r}, 1 \leqq r<2$. For precisely this reason we cannot expect the indices of $\hat{X}$ to exceed $1 / 2$ whenever $X$ has indices equal to $p^{-1}, 2<p \leqq \infty$. In fact, we shall see in $\S 7$ that the following result holds.

TheOREM 6.7. Let $X$ be a rearrangement-invariant space on $T$. If $X \subseteq L^{21}$ (hence certainly if $X$ has indices $\left.p^{-1}, 2<p \leqq \infty\right)$, then $\hat{X}=l^{2 \infty}$, with equivalent norms, and so $\alpha(\hat{X})=\beta(\hat{X})=1 / 2$.

7. Examples. We give a brief description of the space $\hat{X}$ when 
$X$ is a Lorentz space ${ }^{3}$. In particular, we show that the results of Hardy-Littlewood and Calderón mentioned in $\S 1$ are contained in ours as special cases.

(1). If $X=L^{p r}, 1<p<2,1 \leqq r \leqq \infty$, then $\hat{X}_{0}=\hat{X}=l^{q r}$, $p^{-1}+q^{-1}=1$. Indeed, if $g \in l^{q r}$, then $f(t)=f^{*}(t)=t^{-1} g^{* *}\left(t^{+1}\right), 0<t<1$, belongs to $L^{p r}$ and

$$
g^{* *}(s) \leqq s^{-1} f^{* *}(s)=\int_{0}^{1 / s} f^{*}(u) d u \leqq\left(S f^{*}\right)^{* *}(s) .
$$

Hence, by (4.3), $g \in \hat{X}_{0}$ and so $l^{q r} \cong \hat{X}_{0}$. Conversely, if $g \in \hat{X}_{0}$ and $f \in L^{p r}$ satisfies $g^{* *} \leqq\left(S f^{*}\right)^{* *}$, it follows from Hardy's inequality (cf. [4], Chap. I) that $\|g\|_{l^{q r}} \leqq c\|f\|_{L^{p r}}$. Hence, by (4.4), $g \in l^{q r}$ and $\|g\|_{l^{q r}} \leqq\|g\|_{\hat{X}_{0}}$. This shows that $\hat{X}_{0}=l^{q r}$ with comparable, hence equivalent, norms. Finally, since $l^{q r}$ has the Fatou property, it follows from Theorem 5.1 that $\hat{X}_{0}=\hat{X}=l^{q r}$.

(2) If $X=L^{1}$, then $\hat{X}_{0}=c_{0}$ and $\hat{X}=l^{\infty}$. This follows in much the same way as above upon observing that there are functions $f$ in $L^{1}$ for which $\int_{0}^{1 / t} f^{*}(s) d s$ tends to zero arbitrarily slowly as $t \rightarrow \infty$. Note that $c_{0}$ does not have the Fatou property. Indeed, $c_{0}$ is generated by the function norm $\rho(f)=\|f\|_{\infty}, f \in c_{0}, \rho(f)=\infty, f \notin c_{0}$. Thus, if $\chi_{n}$ is the characteristic function of the set $\{1,2, \cdots, n\}$ and $\chi$ is identically equal to 1 on $Z^{+}$, we have $\chi_{n} \uparrow \chi$ pointwise but $\rho\left(\chi_{n}\right)=1$ for all $n$ while $\rho(\chi)=\infty$. The space $\hat{X}=l^{\infty}$ of course has the Fatou property.

(3) When $X=L^{2}$, Theorem 6.6 fails to reproduce the Plancherel theorem, i.e., $\hat{X} \neq l^{2}$. This is of course due to the weak-type behavior of the Calderón operator $S$ (cf. Lemma 3.2). Thus, at least as far as the Hausdorff-Young theorem is concerned, our results for spaces of index $1 / 2$ are uninteresting and will not be pursued here.

(4) If $X \subseteq L^{21}$ then $\hat{X}=\hat{X}_{0}=l^{2 \infty}$. For if $X \subseteq L^{21}$, then

$$
\int_{1 / t}^{1} s^{-1 / 2} f^{*}(s) d s \leqq c_{1}\|f\|_{L^{21}} \leqq c_{2}\|f\|_{X}
$$

and so $\left(S f^{*}\right)^{* *}(t)$ decays as $t^{-1 / 2}$ as $t \rightarrow \infty$. Moreover, this rate of decay is always attained (take $f=$ constant). Thus, arguing as in example (1) above, we see that $\hat{X}_{0}$ and hence $\hat{X}$ coincides with $l^{2 \infty}$, with equivalent norms.

8. The space $\check{Y}$. Let $Y$ be a rearrangement-invariant space on the integers $Z$. We wish to construct a rearrangement-invariant space $\breve{Y}$ on $T$ such that $\mathscr{T}$ is bounded from $\breve{Y}$ into $Y$. Thus we define $Y$ to be the collection of all functions $f$ on $T$ for which $\left(S f^{*}\right)^{* *} \leqq g^{* *}$, for some $g \in Y$, and we set

${ }^{3}$ For the definition of Lorentz spaces, see [3] or [4], Chap. I. 


$$
\|f\|_{\check{Y}}=\inf \left\{\|g\|_{Y}:\left(S f^{*}\right)^{* *} \leqq g^{* *}\right\},
$$

$f \in \check{Y}$.

We remarked in the preceding section that $\left(S f^{*}\right)^{* *}$ cannot decay faster then $t^{-1 / 2}$ so in order that $\bar{Y}$ contain the constant functions we need to know that there are functions $g$ in $Y$ such that $g$ decays more slowly that $t^{-1 / 2}$. This will be the case if, for instance, the indices of $Y$ are equal to $q^{-1}, 2<q \leqq \infty$, because then $l^{2 \infty} \cong Y$.

THEOREM 8.1 (Theorem B). Let $Y$ be a rearrangement-invariant space on $Z$ with indices $\left(q^{-1}, q^{-1}\right), 2<q \leqq \infty$. Then the space $\check{Y}$ is a rearrangement-invariant space on $T$ with indices $\left(p^{-1}, p^{-1}\right), p^{-1}+q^{-1}=$ 1 , and $\mathscr{T}$ is a bounded linear operator from $\breve{Y}$ into $Y$.

Proof. The properties $(2.4), \cdots,(2.8)$ for $\check{Y}$ are established in much the same way as for the space $\hat{X}_{0}$ in $\S 4$; we prove only the triangle inequality (2.6).

Thus, if $f_{1}, f_{2} \in \check{Y}$, then given $\varepsilon>0$ there are functions $g_{i} \in Y$ such that $\left(S f_{i}^{*}\right)^{* *} \leqq g_{i}^{* *}$ and $\left\|g_{i}\right\|_{Y} \leqq\left\|f_{i}\right\|_{Y}+\varepsilon / 2, i=1,2$. From Lemma 3.2 we have

$$
\left(S\left(f_{1}+f_{2}\right)^{*}\right)^{* *} \leqq\left(S f_{i}^{*}\right)^{* *}+\left(S f_{2}^{*}\right)^{* *} \leqq g_{1}^{* *}+g_{2}^{* *}=\left(g_{1}^{*}+g_{2}^{*}\right)^{* *},
$$

so from (8.1) we deduce that $f_{1}+f_{2} \in \check{Y}$ and

$$
\begin{gathered}
\left\|f_{1}+f_{2}\right\|_{\check{Y}} \leqq\left\|g_{1}^{*}+g_{2}^{*}\right\|_{Y} \leqq\left\|g_{1}^{*}\right\|_{Y}+\left\|g_{2}^{*}\right\|_{Y} \\
\quad=\left\|g_{1}\right\|_{Y}+\left\|g_{2}\right\|_{Y} \leqq\left\|f_{1}\right\|_{\check{Y}}+\left\|f_{2}\right\|_{\check{Y}}+\varepsilon .
\end{gathered}
$$

Since $\varepsilon$ is arbitrary, this shows that $\left\|f_{1}+f_{2}\right\|_{\check{Y}} \leqq\left\|f_{1}\right\|_{\check{Y}}+\left\|f_{2}\right\|_{\check{Y}}$, as desired.

Note that the infimum in (8.1) is attained. Indeed, if $f \in \breve{Y}$ then $\|f\|_{\check{Y}}=\left\|g_{f}\right\|_{Y}$ where $g_{f}=g_{f}^{*}$ is the unique function in $Y$ satisfying $\left(g_{f}\right)^{* *}(m)=\left(S f^{*}\right)^{* *}(m-1), m \in Z^{+}$. Thus, if $f_{n} \uparrow f$, we have $\left(S f_{n}^{*}\right)^{* *} \uparrow$ $\left(S f^{*}\right)^{* *}$ and hence $g_{f_{n}} \uparrow g_{f}$. Since $Y$ has the Fatou property we have $\left\|f_{n}\right\|_{\check{Y}}=\left\|g_{f_{n}}\right\|_{Y} \uparrow\left\|g_{f}\right\|_{Y}=\|f\|_{\check{Y}}$, and hence $\breve{Y}$ also has the Fatou property.

The proof of the boundedness of $\mathscr{T}$ and the computation of the indices are much the same as before so we omit the details.

9. Extensions. The preceding theory extends fairly easily to more general groups but one or two remarks are in order. If $G$ is a locally compact abelian group with dual group $\Gamma$ (we assume that the Haar measures are $\sigma$-finite) then the Fourier transform $\mathscr{T}_{G}$ defined on $\left(L^{1}+L^{2}\right)(G)$ is bounded from $L^{1}(G)$ into $L^{\infty}(\Gamma)$ and from $L^{2}(G)$ into $L^{2}(\Gamma)$; hence, if $X$ is a rearrangement-invariant space on $G$ with indices $\left(p^{-1}, p^{-1}\right), 1<p<2$, then $X \subseteq\left(L^{1}+L^{2}\right)(G)$ and so $\mathscr{T}_{G}$ maps $X$ into $\left(L^{2}+L^{\infty}\right)(\Gamma)$. 
Once these properties of $\mathscr{T}_{G}$ have been noted, the group structure is no longer needed. The space $\hat{X}_{0}$ is constructed as before and the computation of the indices is the same. We define $\hat{X}$ by means of the norm $\|g\|_{\hat{X}}=\sup \left\|g \chi_{E_{n}}\right\| \hat{x}_{0}$, where the supremum is taken over all sequences $\left\{E_{n}\right\}_{n=1}^{\infty}$ of sets $E_{n}$ of finite measure such that $E_{n} \uparrow \Gamma$. Of course, if $\Gamma$ has finite measure then $\hat{X}_{0}$ and $\hat{X}$ are identical, but if $\Gamma$ has infinite measure then the example $X=L^{1}$, in which case $\hat{X}$ is $L^{\infty}(\Gamma)$ and $\hat{X}_{0}$ is the closure in $L^{\infty}(\Gamma)$ of functions of compact support, shows that $\hat{X}$ and $\hat{X}_{0}$ need not coincide (cf. $\S 7$, Ex. (2)). Similar remarks apply to the construction of the space $\hat{Y}$.

Note that Theorems A and B are not special to the Fourier transform $\mathscr{T}$; they are valid for any operator of weak types $(1, \infty)$ and $(2,2)$. In the same vein, we remark that Theorems $A$ and $B$ have obvious analogues for operators of weak types $\left(p_{0}, p_{1}\right)$ and $\left(q_{0}, q_{1}\right), 1 \leqq p_{i}, q_{i} \leqq \infty$.

\section{REFERENCES}

1. C. Bennett and J. E. Gilbert, Harmonic analysis of some function spaces on $T^{n}$, (to appear).

2. D. W. Boyd, Indices of function spaces and their relationship to interpolation, Canad. J. Math., 21 (1969), 1245-1254.

3. A. P. Calderón, Spaces between $L^{1}$ and $L^{\infty}$ and the theorem of Marcinkiewicz, Studia Math., 26 (1966), 273-299.

4. J. E. Gilbert and C. Bennett, Interpolation Space Theory and Harmonic Analysis, (lecture notes in preparation).

5. G. H. Hardy and J. E. Littlewood, Some new properties of Fourier constants, Math. Ann., 97 (1926), 159-209.

6. Notes on the theory of series (XIII): Some new properties of Fourier constants, J. London Math. Soc., 6 (1931), 3-9.

7. W. A. J. Luxemburg, Rearrangement-invariant Banach function spaces, Queen's Papers in Pure and Applied Mathematics, 10 (1967), 83-144, Queen's University, Canada. 8. W. A. J. Luxemburg and A. C. Zaanen, Notes on Banach function spaces, Notes I-V, Proc. Acad. Sci. Amsterdam, A 66 (Indag. 25), 135-147, 148-153, 239-250, 251-263, 496-504 (1963).

9. A. Zygmund, Trigonometric Series, Vol. II, Cambridge University Press, Cambridge, 1959.

Received April 5, 1972.

California Institute of Technology 


\section{PACIFIC JOURNAL OF MATHEMATICS}

\section{EDITORS}

D. Gilbarg AND J. MILGRAM

Stanford University

Stanford, California 94305

\section{R. A. Beaumont}

University of Washington

Seattle, Washington 98105

\section{J. DUGUNDJI*}

Department of Mathematics University of Southern California Los Angeles, California 90007

RICHARD ARENS

University of California Los Angeles, California 90024

\section{ASSOCIATE EDITORS}
E. F. BECKENBACH
B. H. NEUMANN
F. WOLF
K. YoshidA

\section{SUPPORTING INSTITUTIONS}

\author{
UNIVERSITY OF BRITISH COLUMBIA \\ CALIFORNIA INSTITUTE OF TECHNOLOGY \\ UNIVERSITY OF CALIFORNIA \\ MONTANA STATE UNIVERSITY \\ UNIVERSITY OF NEVADA \\ NEW MEXICO STATE UNIVERSITY \\ OREGON STATE UNIVERSITY \\ UNIVERSITY OF OREGON \\ OSAKA UNIVERSITY
}

\author{
UNIVERSITY OF SOUTHERN CALIFORNIA \\ STANFORD UNIVERSITY \\ UNIVERSITY OF TOKYO \\ UNIVERSITY OF UTAH \\ WASHINGTON STATE UNIVERSITY \\ UNIVERSITY OF WASHINGTON \\ AMERICAN MATHEMATICAL SOCIETY \\ NAVAL WEAPONS CENTER
}

The Supporting Institutions listed above contribute to the cost of publication of this Journal, but they are not owners or publishers and have no responsibility for its content or policies.

Mathematical papers intended for publication in the Pacific Journal of Mathematics should be in typed form or offset-reproduced, (not dittoed), double spaced with large margins. Underline Greek letters in red, German in green, and script in blue. The first paragraph or two must be capable of being used separately as a synopsis of the entire paper. Items of the bibliography should not be cited there unless absolutely necessary, in which case they must be identified by author and Journal, rather than by item number. Manuscripts, in duplicate if possible, may be sent to any one of the four editors. Please classify according to the scheme of Math. Rev. Index to Vol. 39. All other communications to the editors should be addressed to the managing editor, Richard Arens, University of California, Los Angeles, California, 90024.

50 reprints are provided free for each article; additional copies may be obtained at cost in multiples of 50 .

The Pacific Journal of Mathematics is issued monthly as of January 1966. Regular subscription rate: $\$ 48.00$ a year (6 Vols., 12 issues). Special rate: $\$ 24.00$ a year to individual members of supporting institutions.

Subscriptions, orders for back numbers, and changes of address should be sent to Pacific Journal of Mathematics, 103 Highland Boulevard, Berkeley, California, 94708.

PUBLISHED BY PACIFIC JOURNAL OF MATHEMATICS, A NON-PROFIT CORPORATION

Printed at Kokusai Bunken Insatsusha (International Academic Printing Co., Ltd.), 270, 3-chome Totsuka-cho, Shinjuku-ku, Tokyo 160, Japan.

* C. DePrima will replace J. Dugundji until August 1974.

Copyright (C) 1973 by

Pacific Journal of Mathematics

All Rights Reserved 


\section{Pacific Journal of Mathematics}

\section{Vol. 47, No. $2 \quad$ February, 1973}

David Parham Bellamy, Composants of Hausdorff indecomposable continua; a mapping approach ........................ 303

Colin Bennett, A Hausdorff-Young theorem for rearrangement-invariant spaces ...........................................

Roger Daniel Bleier and Paul F. Conrad, The lattice of closed ideals and $a^{*}$-extensions of an abelian l-group ...

Ronald Elroy Bruck, Jr., Nonexpansive projections on subsets of Banach

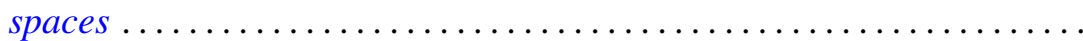

Robert C. Busby, Centralizers of twisted group algebras ............. 357

M. J. Canfell, Dimension theory in zero-set spaces ................ 393

John Dauns, One sided prime ideals ........................ 401

Charles F. Dunkl, Structure hypergroups for measure algebras . . . . . . . . . 413

Ronald Francis Gariepy, Geometric properties of Sobolev mappings ...... 427

Ralph Allen Gellar and Lavon Barry Page, A new look at some familiar spaces of intertwining operators ...........................

Dennis Michael Girard, The behavior of the norm of an automorphism of the

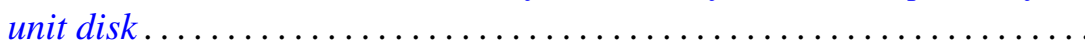

George Rudolph Gordh, Jr., Terminal subcontinua of hereditarily

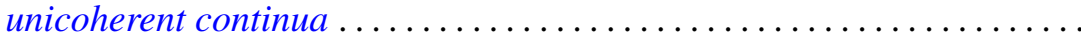

Joe Alston Guthrie, Mapping spaces and cs-networks. .

Neil Hindman, The product of $F$-spaces with $P$-spaces . 473

M. A. Labbé and John Wolfe, Isomorphic classes of the spaces $C_{\sigma}(S)$

Ernest A. Michael, On k-spaces, $k_{R}$-spaces and $k(X) \ldots$

Donald Steven Passman, Primitive group rings .

C. P. L. Rhodes, A note on primary decompositions of a pseudovaluation ...

Muril Lynn Robertson, A class of generalized functional differential equations

Ruth Silverman, Decomposition of plane convex sets. $I$.

Ernest Lester Stitzinger, On saturated formations of solvable Lie algebras................................

B. Andreas Troesch, Sloshing frequencies in a half-space by Kelvin inversion ...

L. E. Ward, Fixed point sets .

Michael John Westwater, Hilbert transforms, and a problem in scattering

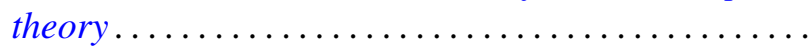

Misha Zafran, On the spectra of multipliers... 